\title{
Melting of Crystals in Two Dimensions
}

\author{
Urs Gasser, ${ }^{*[a]}$ Christoph Eisenmann, ${ }^{[b]}$ Georg Maret, $^{[b]}$ and Peter Keim ${ }^{[b]}$
}

While the melting of crystals is in general not understood in
detail on a microscopic scale, there is a microscopic theory for
a class of two-dimensional crystals, which is based on the for-
mation and unbinding of topological defects. Herein, we
review experimental work on a colloidal two-dimensional
model system with tunable interactions that has given the first conclusive evidence for the validity of this theory on a microscopic level. Furthermore, we show how the mechanism of melting depends on the particle interaction and that a strong anisotropy of the interaction leads to a changed melting scenario.

\section{Introduction}

Like other phase transitions, the melting of crystals is a central topic in condensed-matter physics and is of interest for the understanding of all crystalline materials. Freezing and melting have been studied for centuries, which reflects their broad relevance for many fields. Nevertheless, a detailed microscopic understanding of the melting transition is lacking for most materials, although a microscopic theory for the melting of crystals in two dimensions (2D) has been developed in the 1970s by Kosterlitz, Thouless, Halperin, Nelson, and Young (KTHNY theory). ${ }^{[1-6]}$ It explains crystal melting by the dissociation of pairs of defects that form close to the melting point.

One reason for the lack of microscopic theories for melting is the difficulty in observing the transition of atomic or molecular materials on the microscopic scale of the constituents. Therefore, colloidal suspensions are increasingly used as model systems for the study of melting and other phase transitions, since they show phase behavior that is analogous to that of atomic materials and, furthermore, the size of colloidal particles and their interactions can be tailored for experiment.

Herein we focus on KTHNY theory and experiments that first gave experimental evidence for its validity. This article is organized as follows. A general introduction of essential properties of $2 \mathrm{D}$ crystals and the KTHNY theory are presented in Section 2 and early experiments on melting in $2 \mathrm{D}$ are presented in Section 3. The colloidal model system from which conclusive evidence for the KTHNY melting scenario has been obtained and the corresponding experiments carried out with this system are presented in Sections 4 and 5. A study of the interactions between dislocations in 2D crystals is reviewed in Section 6 and the effects of an anisotropic particle-interaction imposed by an external field are presented in Section 7 .

\section{2D Crystals and KTHNY Theory}

As for most crystal properties, the melting transition in 2D differs from that in $3 D$, because the reduced dimensionality prevents the long-range translational order of $3 \mathrm{D}$ crystals. This is due to long-wavelength fluctuations that are easily excited in $1 D$ and 2D, while their energy diverges with the wavelength in
3D. This was first shown by Peierls for the magnetic $X Y$ system. ${ }^{[7]}$ In a ferromagnetic material, the tilting of a spin by a small angle $\delta \phi$ with respect to its neighbors takes an energy $\left(E \propto \delta \phi^{2}\right)$. The excitation of a spin-wave with wavelength $L$ then requires an energy $\propto L^{\mathrm{d}} \delta \phi^{2} \propto L^{\mathrm{d}}(2 \pi / L)^{2}$, where $d \in\{1,2,3\}$ is the dimensionality. This energy diverges with $L$ for $d=3$, while for $d=2$ it is independent of $L$ and for $d=1$ it decreases $\propto L^{-1}$. Therefore, the crystal does not exist for $d=1$ and for $d=2$ its translational order is reduced to quasi-long-range. ${ }^{[6]}$

Even today, the melting transition of most materials is not well understood, because theories explaining the transition on a microscopic scale are not available. Furthermore, the mechanism of melting depends on the details of the interactions between the particles forming the crystal lattice and defects reducing the translational order of the crystal are expected to play a mayor role. For example, as observed in 3D crystals of microgel particles, ${ }^{[8]}$ the melting transition was found to start at grain boundaries. There are several theories for the melting transition in 2D. The formation of grain boundaries ${ }^{[9-11]}$ as well as the condensation of dislocation ${ }^{[12]}$ have been put forward as possible mechanisms. In the 1970s, a theory based on the formation of topological defects was developed by Kosterlitz, Thouless, Halperin, Nelson, and Young (KTHNY theory). ${ }^{[1,2,4-6]}$ Therefore for the class of $2 \mathrm{D}$ crystals where this theory applies, melting of $2 \mathrm{D}$ crystals is understood in more detail than in $3 \mathrm{D}$.

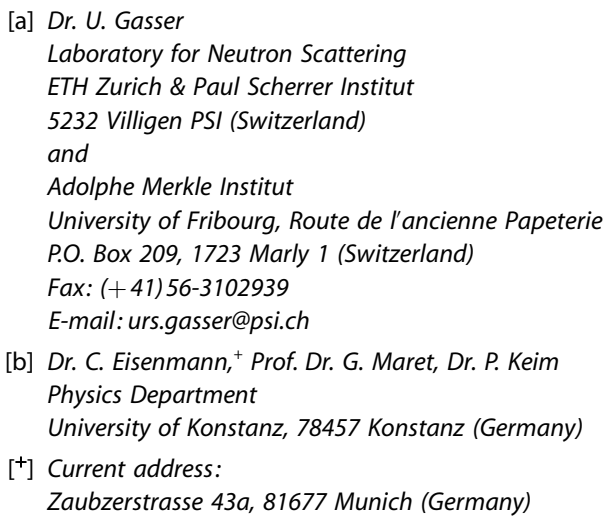

[a] Dr. U. Gasser

Laboratory for Neutron Scatte

and

Adolphe Merkle Institut

Fax: $(+41) 56-3102939$

E-mail: urs.gasser@psi.ch

Physics Department

University of Konstanz, 78457 Konstanz (Germany)

Zaubzerstrasse 43a, 81677 Munich (Germany) 
The applicability of the KTHNY theory depends on the interaction between the particles. During recent years conclusive proofs for its validity have been presented.

According to this theory, melting is based on the decoupling of pairs of topological defects and it predicts the existence of an intermediate equilibrium phase-the hexatic phase-between the crystal and the liquid state, which is specific to melting due to topological defects in 2D. The transitions from the crystal to the hexatic phase and further to the liquid are predicted to be continuous. Bound pairs of dislocations can form in the crystalline state as illustrated in Figure $1 \mathrm{~A}$. Particles are

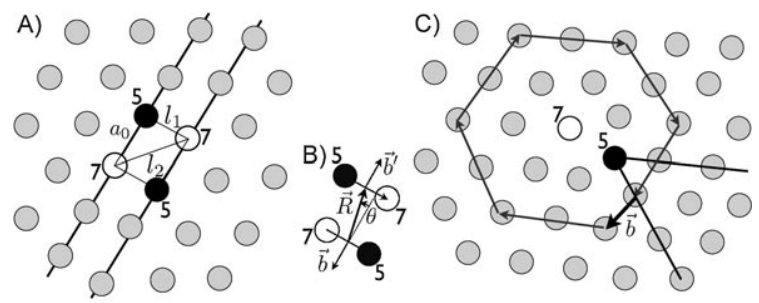

Figure 1. A) A pair of dislocations at minimum distance, which can be formed by displacing the particles on the lattice lines marked by the black lines. $l_{1}=\sqrt{2 a_{0} / 2}$ and the distance of $I_{2}=\sqrt{7 a_{0} / 2}$ between the sevenfold coordinated particles corresponds to the maximum distance for the seven particles to be neighbors. B) Burgers vectors $\vec{b}$ and the vector $\vec{R}$ giving the orientation of the dislocation pair are shown. C) The orientation of a dislocation is given by the Burgers vector $\vec{b}$, which is obtained from a track around the dislocation with edges comprising a constant number of particles (gray arrows). The lattice lines ending at the fivefold coordinated particle of the dislocation are indicated by the black lines.

displaced such that two have seven instead of the normal six nearest neighbors and for two of their neighbors the number is reduced to five. A pair of five- and sevenfold coordinated particles form a dislocation, which distorts the crystal lattice and, therefore, is an improbable event in the crystal phase. An isolated dislocation is characterized by the Burgers vector giving the orientation of the dislocation (Figure $1 \mathrm{C}$ ). The translational order is decreased in the direction given by the Burgers vector. In the case of a triangular crystal, a dislocation can be viewed as two extra lattice lines that end at the fivefold coordinated particle. As a consequence, it is a topological defect that cannot form in the crystal from particle displacements in a finite region, costing a finite amount of free energy and destroying the translational order of the crystal. However, dislocation pairs with antiparallel extra lattice lines correspond to a structural change in a limited region of the crystal and can form spontaneously if the involved free energy is not too high. As the crystal lattice is distorted over an increasingly large area, the elastic energy needed for this separation between two dislocations grows the farther they are located from each other. Dislocations, therefore, interact with each other attractively. The Hamiltonian of this interaction was originally developed for crystals in $3 D^{[13]}$ and, for dislocations in $2 D$, reduces to the form given by Equation (1):
$H_{\mathrm{D}}=-\frac{Y a_{0}^{2}}{4 \pi}\left\{\vec{b}(\vec{r}) \cdot \vec{b}^{\prime}\left(\vec{r}^{\prime}\right) \log \frac{R}{a_{\mathrm{c}}}-\frac{[\vec{b}(\vec{r}) \cdot \vec{R}]\left[\vec{b}^{\prime}\left(\vec{r}^{\prime}\right) \cdot \vec{R}\right]}{R^{2}}\right\}+2 E_{\mathrm{c}}$

with $a_{0}$ the lattice constant and $a_{c}$ the dislocation core diameter. $\vec{b}$ represents the Burgers vector of a dislocation and $E_{c}$ is the core energy of a dislocation, ${ }^{[14]}$ which is introduced to account for the energy needed to create the core region of a dislocation given by a five- and sevenfold coordinated particle. This energy is not given by the first term of Equation (1), which tends to diverge for $R$ smaller than $a_{\mathrm{c}}$ and, therefore, is only valid when $R$ is large enough. The coupling constant of $H_{\mathrm{D}}$ is essentially given by Young's modulus $Y$ measuring the rigidity of the crystal lattice. $H_{\mathrm{D}}$ is a cornerstone of KTHNY theory, which determines the elastic constants of the crystal in the presence of defects with a renormalization procedure. ${ }^{[2]}$ KTHNY predicts that 2D crystals melt due to the formation of dislocation pairs in the crystalline state close below the melting temperature $T_{\mathrm{m}}$ and to the unbinding of such pairs at the transition. The unbinding destroys the translational order and involves a large increase of elastic free energy due to the large-scale distortion involved in the formation of an isolated dislocation. It can be shown that the elastic energy due to an isolated dislocation or disclination is proportional to $\log r / a_{\mathrm{c}}$ for large enough distances $r$ from the center of the defect. Here, $a_{c}$ is the core size of the defect, which is of the order of the nearest-neighbor distance. Furthermore, the crystal becomes mechanically weaker due to the appearance of isolated dislocations-the value of the Young's modulus $Y$ is reduced and KTHNY predicts that the dimensionless quantity $Y a_{0}^{2} /\left(k_{\mathrm{B}} T\right)$ reaches a value of $16 \pi$ at melting. The translational order of the crystal is measured by the correlation function given by Equation (2):

$G_{T}(|\vec{r}-\vec{r}|)=\left\langle e^{-i \vec{G} \cdot(\vec{r}-\vec{r})}\right\rangle$

where $\vec{G}$ is a reciprocal lattice vector and the angled brackets on the right hand side denote an average over particles at positions $\vec{r}$ and $\vec{r}$ with distance $|\vec{r}-\vec{r}| . G_{T}(r) \propto r^{-\eta_{T}}$ due to the quasi-long-range translational order of the crystal and in the hexatic as well as the liquid state it decays exponentially. Another parameter that is useful for localizing the melting transition is the Lindemann parameter $\gamma_{\mathrm{L}}(t){ }_{1}^{[15]}$ which measures the mean-square deviation of a particle. It stays finite in the crystal, but diverges in the liquid phase. Due to the quasi-long-range order of $2 \mathrm{D}$ crystals, a modified Lindemann parameter has to be used, which measures the particle displacement relative to the nearest neighbors [Eq. (3)]:[16,17]

$\gamma_{\mathrm{L}}(t)=\left\langle\left[\Delta r_{j}(t)-\Delta r_{j+1}(t)\right]^{2}\right\rangle /\left(2 a^{2}\right)$

with $\Delta r_{j}(t)=r_{j}(t)-r_{j}(0)$ and $a$ is the lattice constant. In 2D, $\gamma_{L}(t)$ is expected to reach a value of 0.033 upon melting. ${ }^{[17]}$

The melting transition at $T_{\mathrm{m}}$ reduces but does not entirely destroy the orientational order, which is measured by the orientational correlation function given by Equation (4) 
$G_{6}(|\vec{r}-\vec{r}|)=\left\langle q_{6}(\vec{r}) q_{6}(\vec{r})\right\rangle$

where [Eq. (5)]:

$q_{6}\left(\vec{r}_{j}\right)=\frac{1}{N} \sum_{K=1}^{N} e^{-6 i \theta_{j k}}$

and where $q_{6}$ measures the orientation of the $N$ neighbors of particle $j$. With the factor six in the exponent, $\left|q_{6}\right|$ can reach a value of one in the hexagonal lattice, while defects and disorder reduce the value. The orientational order is reduced from long-range to quasi-long-range due to the presence of the isolated dislocations. Thus, $G_{6}(r)$ approaches a constant value $>0$ in the crystal, decays algebraically in the hexatic $\left(\propto r^{-\eta 6}\right)$, and exponentially in the liquid state. The second transition from the hexatic to the liquid state happens at a higher temperature $T_{\mathrm{i}}$ due to the unbinding of disclinations into isolated disclinations. These are isolated five- or sevenfold coordinated particles, which are another type of topological defect and further reduce the orientational order from quasi-long-range to shortrange (Figure 2). The orientational order corresponds to a stiff-

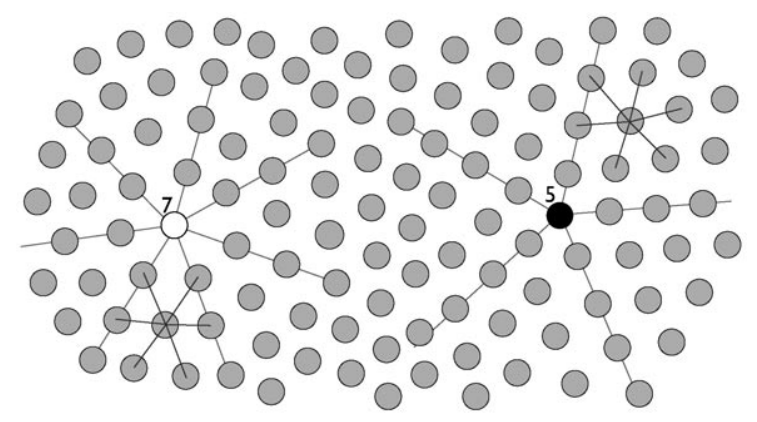

Figure 2. Two disclinations formed by a five- and sevenfold coordinated particle as illustrated by the gray lines. The orientational order changes close to disclinations as shown for two particles by the dark gray lines.

ness of the system with respect to rotational shear, which is measured by Frank's constant $F_{\mathrm{A}}$ known from liquid crystals. ${ }^{[18]}$ In analogy to the interaction of dislocations, the coupling constant of the interaction between disclinations is given by $F_{\mathrm{A}}$. According to $\mathrm{KTHNY}, F_{\mathrm{A}} /\left(k_{\mathrm{B}} T\right)$ reaches a value of $72 / \pi$ at $T=T_{\mathrm{i}}$.

\section{Early Experiments and Computer Simulations}

Conclusive evidence for the existence of the hexatic phase and two continuous transitions for melting in 2D was missing for a long time after the development of the KTHNY theory. The main difficulties that were encountered in experiments and simulations were related to the structural change from crystal to liquid not being as clear as in $3 \mathrm{D}^{[19]}$ and to difficulties in distinguishing between the hexatic phase and crystal-liquid coexistence, which is expected for a first-order transition. In early computer simulations, ${ }^{[20-22]}$ no clear conclusions about the existence of the hexatic phase could be reached due to the relatively small number of simulated particles, which limited the reliability of the results. However, recent computer simulations did show the behavior that is expected from KTHNY theory. ${ }^{[23]}$ In many experiments investigating the 2D melting transition, interactions between particles and a substrate defining the dimensionality of the system complicated clear conclusions about the phase transition. The first observations of the hexatic phase were presented by Murray and van Winkle ${ }^{[24]}$ and later by Tang et al., ${ }^{[25]}$ who used a system of charge-stabilized polystyrene spheres confined between glass plates. In a 2D system of particles interacting essentially like hard spheres, the hexatic phase was observed but the transitions from crystal to hexatic and from hexatic to liquid appeared to be of first order. ${ }^{[2]}$ Dislocations and disclinations behaving as expected from KTHNY were observed in a system of polystyrene particles with electric dipole-dipole interactions. ${ }^{[27,28]}$ However, also in that work no conclusive results for or against continuous melting transitions were found. The first clear evidence for the validity of the KTHNY melting scenario came from a colloidal model system of super-paramagnetic particles, which is presented in the following section. ${ }^{[17,29,30]}$

\section{2D Colloidal Model System}

Colloidal particles with a diameter of $4.5 \mu \mathrm{m}$ are suspended in water and are fixed by gravity to the water-air interface of a hanging water droplet. An external magnetic field $\vec{B}$ is applied perpendicular to or somewhat tilted by an angle $\varphi$ relative to the particle plane. As the particles are super-paramagnetic due to doping with $\mathrm{Fe}_{2} \mathrm{O}_{3}$ nanoparticles, the field induces a magnetic moment $\vec{M}=\chi \vec{B}$ in each particle, where $\chi$ is the magnetic susceptibility. The resulting dipole-dipole interaction is repulsive and $\propto 1 / r^{3}$ when the field is perpendicular to the particle plane [Eq. (6)]:

$u(r)=\frac{\mu_{0}(\chi B)^{2}}{8 \pi} \frac{1}{r^{3}}$

An anisotropic interaction is obtained for a tilting $\varphi>0^{\circ}$ of the field: Along an in-plane component of the magnetic field, the interaction is less repulsive, which is reflected in the structure formed by the particles. The dipole-dipole interaction in this system dominates all other interactions (charge, steric repulsion), which therefore need not be taken into account. ${ }^{[31,32]}$ Furthermore, the suspension contains a small amount of SDS surfactant, which covers the particles and leads to complete wetting. As a consequence, the deformation of the water-air surface due to the particles is minimal and can also be neglected. The particles are observed by video microscopy and their coordinates are determined in real time. $\sim 2000$ particles were usually in the field of view with a size of $850 \times 650 \mu \mathrm{m}^{2}$, while the whole cell with a diameter of $8 \mathrm{~mm}$ contains roughly $3 \times 10^{5}$ particles. The apparent size of the particles depends on their height relative to the focal plane of the microscope and is used to correct the flatness of the water droplet with a precision of $1 \mu \mathrm{m}$ by adding or removing small amounts of water with a motorized syringe. ${ }^{[31]}$ Thus, the particle density is kept homogenous. For $\varphi=0^{\circ}$ and at a low effective temperature, hexagonal crystals without defects can be obtained, as 
shown in Figure 3. This indicates that the (magnetic) polydispersity of the particles is low.

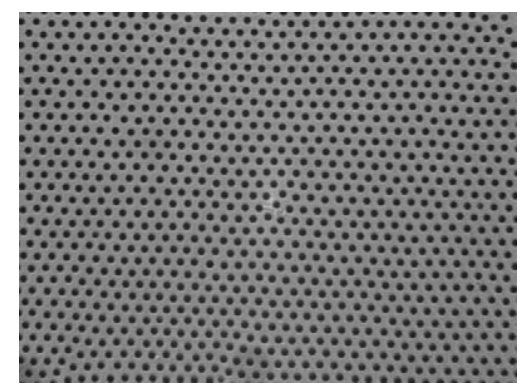

Figure 3. Microscopy image of a 2D colloidal crystal with triangular lattice and isotropic dipole-dipole repulsion between the particles.

As the interaction strength is controlled by the applied magnetic field, the effective temperature of the system can be changed by varying $B$. The effective inverse system temperature is defined by Equation (7):

$\Gamma=\frac{E_{\mathrm{p}}}{k_{\mathrm{B}} T}=\frac{\mu_{0}(\chi B)^{2}}{8 \pi k_{\mathrm{B}} T} \rho^{3 / 2}(2 \cos \psi \sin \psi)^{3 / 2} C_{\text {Madelung }}(\varphi, \psi)$

where $\rho$ is the area-density of the particles and [Eq. (8)]:

$C_{\text {Madelung }}(\varphi, \psi)=\sum_{j} \frac{r_{i j}^{2}-3\left(\hat{B} \cdot \vec{r}_{i j}\right)^{2}}{r_{i j}^{5}}$

is the Madelung constant of the crystal structure formed by the particles. The Madelung constant has to be taken into account when measurements with isotropic and anisotropic interaction are compared, since the structure and the interaction energy depend on the tilting angle $\varphi . \psi$ gives the distortion of the crystal lattice as explained in Section 7. In work where only isotropic interactions were considered, the Madelung constant was not taken into account. For this reason, the effective temperatures $\Gamma$ given herein differ from those in previous publications. ${ }^{[17,29,30,31,33]}$

\section{Verification of the KTHNY Melting Scenario}

The system was equilibrated and prepared in a monocrystalline state during several days before $\Gamma$ was reduced to investigate the melting transition. After each change of $\Gamma$, the system was left to equilibrate for at least two hours, before particle coordinates were determined. As apparent from the behavior of $G_{6}(r)$ shown in Figure 4, a transition from long-range to quasi-longrange orientational order is observed at $\Gamma_{\mathrm{m}}=48.5 \pm 0.5$, as for lower $\Gamma$ values the orientational correlation function $G_{6}(r)$ does not approach a constant value at large $r$ but is well described by a power-law $G_{6}(r) \propto r^{-\eta_{6}}$. At the same effective temperature the translational order changes from quasi-long-range to short range.

The appearance of defects that cause the crystal to melt can be followed by video microscopy. This supports the KTHNY theory prediction that the crystal indeed melts due to the un-

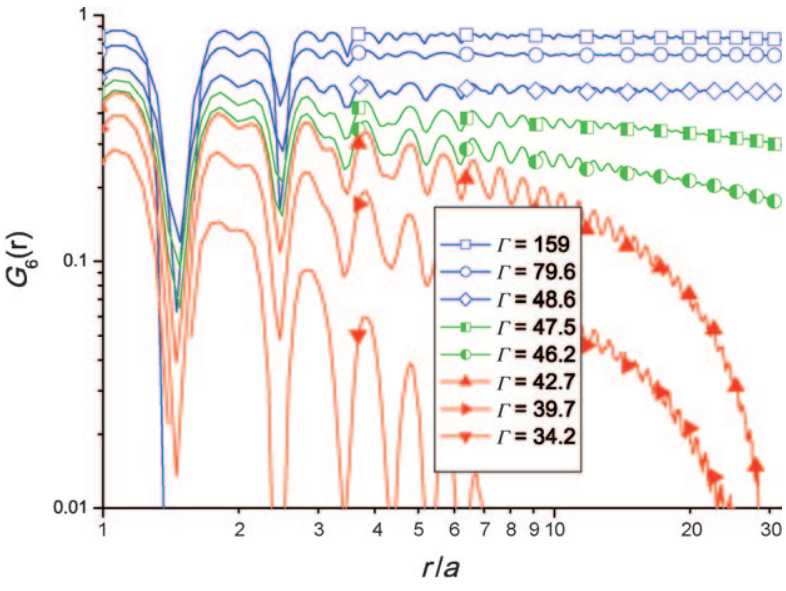

Figure 4. Orientational correlation function $G_{6}(r)$ for different effective temperatures $\Gamma$. Blue refers to the crystalline, green to the hexatic, and red to the liquid state. The oscillations reflect the arrangement of the particles in shells around a central particle at $r=0$. Reprinted with permission from ref. [30]. Copyright 2007 by the American Physical Society.

binding of dislocation pairs that appear close to $\Gamma_{\mathrm{m}}$ in the crystalline state. In Figure $5 \mathrm{~A}$ bound dislocation pairs as well as a few unbound dislocations are visible at $\Gamma=48$ just below the melting point.

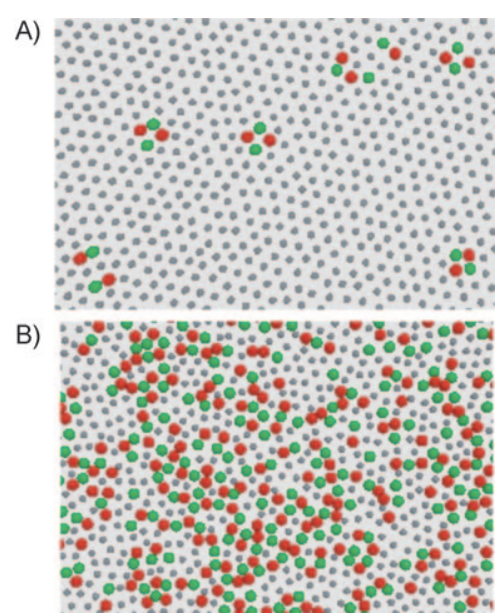

Figure 5. Measured particle configurations $A$ ) just below the melting transition at $\Gamma=48$ and B) in the liquid state. Five- and sevenfold coordinated particles are shown by red and green dots, respectively, while grey dots represent particles with six neighbors. Bound and unbound dislocations are visible in (A), while larger clusters of five- and sevenfold coordinated particles dominate in (B).

As in the case of the melting transition, the transition from the hexatic to the isotropic fluid phase is also found from the behavior of $G_{6}(r)$. According to KTHNY, the quasi-long-range orientational order is lost in the fluid state and $G_{6}(r)$ decays exponentially. As shown in Figure 4, this transition is found at $\Gamma_{i}=45.7 \pm 0.5$, where the exponent of the quasi-long-range decay reaches a value $\eta_{6}=1 / 4$ as expected from KTHNY theory. ${ }^{[17]}$ This transition is caused by the appearance of isolated disclinations (five- or sevenfold coordinated particles), 
which are observed to appear at $\Gamma_{i}$ in the video microscopy experiment (Figure 5B).

KTHNY predicts critical values for the elastic constants at the transitions, which do not depend on the nature of the $2 \mathrm{D}$ system: $Y_{\mathrm{m}} a_{0}^{2} /\left(k_{\mathrm{B}} T\right)=16 / \pi$ and $F_{A, i} /\left(k_{\mathrm{B}} T\right)=72 / \pi$. Therefore, a convincing proof for the KTHNY melting scenario is to show that these critical values are indeed reached at the transitions. $F_{\mathrm{A}}$ can be obtained from the exponent $\eta_{6}(\Gamma)$ in the hexatic phase and $Y$, in principle, from the exponent of $\eta_{\mathrm{T}}(\Gamma)$ in the crystalline phase. Since reciprocal lattice vectors for $G_{\mathrm{T}}(r)$ [Eq. (2)] are not easily determined in $2 \mathrm{D}$ crystals, $^{[34]}$ a method based on the dispersion relation of the crystal normal modes was used. ${ }^{[35]}$ The Young's modulus can be obtained from the $q \rightarrow 0$ behavior of the longitudinal and transversal normal modes. Accordingly, careful measurements of $G_{6}(r)$ that were carried out as explained above ${ }^{[30]}$ and measurements of the crystal normal modes ${ }^{[35]}$ have been used to determine the elastic constants near the phase transitions. As shown in Figure 6,

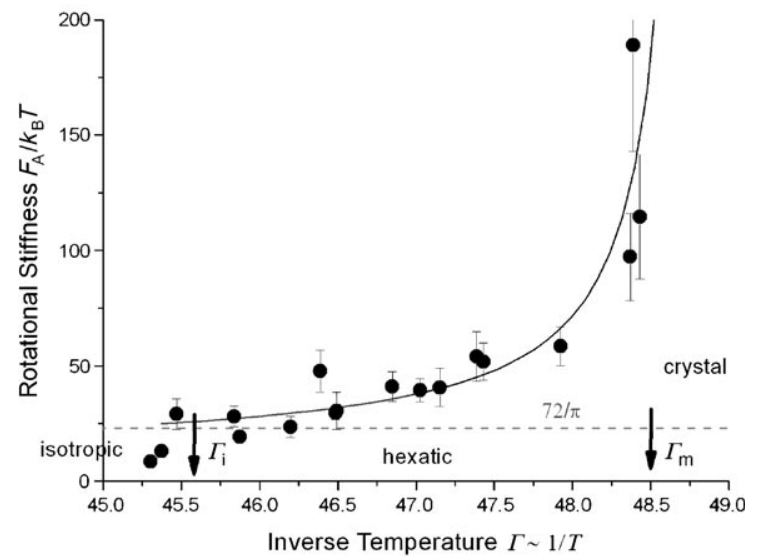

Figure 6. Frank's constant $F_{\mathrm{A}}$ as a function of effective inverse temperature $I$ $(\bullet)$. The curve represents a fit to the measured data and the horizontal dashed line shows the value of $72 / \pi$, which is predicted by KTHNY theory for the transition from hexatic to isotropic liquid. Reprinted with permission from ref. [30]. Copyright 2007 by the American Physical Society.

the Frank constant shows the expected behavior in the hexatic phase: As $\eta_{6}$, it diverges at $\Gamma_{\mathrm{m}}$, because the orientational order becomes long-range. More importantly, the value of $F_{\mathrm{A}}$ approaches $72 / \pi$ near $\Gamma_{i}$ and the experiment indicates a fast drop of $F_{\mathrm{A}}$ for $\Gamma<\Gamma_{i}$, where $F_{\mathrm{A}}$ is expected to vanish. In the crystal state, $Y$ is found to follow the behavior expected from KTHNY theory (Figure 7) ${ }^{[33,36]}$ Moreover, the transitions and behavior of both $G_{6}(r)$ and $G_{T}(r)$ have been found to occur at the same $\Gamma$ for both 'heating' and 'cooling' by varying the magnetic field: No hysteresis is observed, if the system is cooled and heated slowly to keep it in thermal equilibrium. ${ }^{[37]}$ This corroborates the presence of two continuous transitions. The melting transition is, thus, identified from the behavior of $G_{\mathrm{T}}(r)$ on the side of the crystal phase (high- $\Gamma$ side) and from the behavior of $G_{6}(r)$ on the side of the hexatic phase (low- $\Gamma$ side). Both methods agree very well and yield the value $\Gamma_{\mathrm{m}}=48.5 \pm 1.5$.

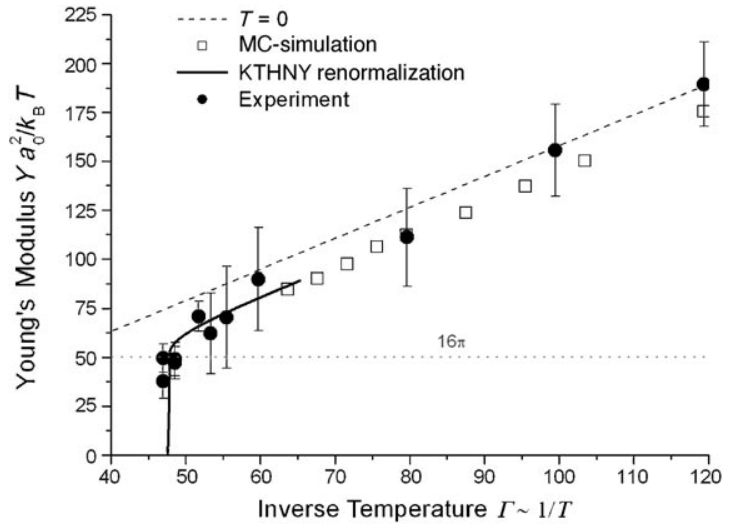

Figure 7. Young's modulus $Y$ as a function of effective inverse temperature $\Gamma$ obtained from experiment $(\bullet)$ and Monte Carlo simulation $(\gamma){ }^{[44]}$ The value of $16 \pi$ that KTHNY theory predicts for the melting transition is marked by the horizontal dashed line; the lines represent the behavior expected for $T=0$ (----) and from KTHNY theory (-). Reprinted with permission from ref. [44]. Copyright 2005 by Institute of Physics Publishing.

\section{Interaction between Dislocations}

From the apparent validity of KTHNY theory as presented in the preceding section, it is expected that the Hamiltonian $H_{\mathrm{D}}$ describing the interaction between dislocations [Eq. (1)] is valid for the defects that are observed in the colloidal model system. However, $H_{\mathrm{D}}$ results from a continuum approximation for the crystalline state and its validity on the microscopic scale of nearest neighbor particles is not evident. The behavior of dislocations found in experiments and Monte Carlo simulations of the crystalline state were, therefore, compared with the expectation from $H_{D}{ }^{[14]}$ Dislocations were identified using the Voronoi construction for measured particle coordinates, which yields the coordination number for each particle. Dislocations are found by looking for two neighboring five- and sevenfold coordinated particles that have sixfold coordinated neighbors. For a quantitative analysis of dislocations, the center is defined as the mid-point of the vector $\vec{r}_{57}$ joining the five- and the sevenfold coordinated particles. The Burgers vector $\vec{b}$ is oriented close to perpendicular to $\vec{r}_{57}$ (see Figure $1 \mathrm{~B}$ ).

The distance dependence of $H_{\mathrm{D}}$ was studied for the case of isolated dislocations with antiparallel Burgers vectors $\vec{b}_{1}=-\vec{b}_{2}$, for which the interaction Hamiltonian has the form given by Equation (9):

$\beta H_{\mathrm{D}}=\beta \frac{Y a_{0}^{2}}{4 \pi}\left[\log \frac{R}{a_{\mathrm{c}}}+0.788\right]$

Because the interaction is attractive, most such dislocations are located at the minimal distance $R / a_{0}=1$. The number distribution $E(R)$ of dislocations at distance $R$ must be normalized by the number of possible dislocation pairs for each distance $R$ to obtain the probability $P(R)$ of formation for a pair at distance $R$. Since $\beta H_{\mathrm{D}}=-\log P(R)$, a direct comparison of experiment and theory is obtained, as shown in Figure 8 . The good agreement at several values of $\Gamma$ in the crystalline phase is only troubled by the relatively small deviations at $R / a_{0} \geq 2$, 


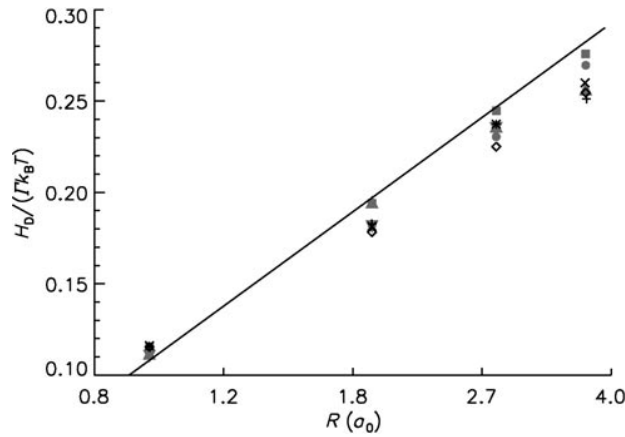

Figure 8. Dependence of the dislocation interaction on distance $R$ for the case of antiparallel Burgers vectors. Experiment: $\Gamma=55.0(+), \Gamma=53.4(\times)$, $\Gamma=51.8(\diamond)$; simulation: $\Gamma=48.6(\backsim), \Gamma=51.0(\diamond), \Gamma=55.7(\triangle), \Gamma=59.7(\nabla)$; (-): prediction according to $H_{\mathrm{D}}$ [Eq. (1)]. Reprinted with permission from ref. [14]. Copyright 2005 by the American Physical Society.

which is due to several factors. Some clusters of dislocations and higher complexions are erroneously not excluded, manybody interactions between close by dislocations are not taken into account, and some dislocations appear due to artifacts such as too small or too big colloidal particles. Furthermore, the $T=0 \mathrm{~K}$ value of $Y$ is used. These factors all lead to a seemingly higher probability for finding two dislocations at some distance $R$

The $\Gamma$-dependence was studied for dislocation pairs with antiparallel Burgers vectors $\vec{b}_{1}=-\vec{b}_{2}$ at the minimum distance $R /$ $a_{0}=1 . H_{\mathrm{D}}$ takes the form shown in Equation (9) with the value for $R$ given above and $Y a_{0}^{2} \beta=1.258 \Gamma$. A comparison of experiments, simulations, and theory is shown in Figure 9, where effective inverse temperatures in the $\Gamma$-range from deep in the crystalline phase down to below the melting point $\Gamma_{\mathrm{m}}=48.5 \pm 0.5$ are shown. The agreement is excellent even below the melting point, which is outside of the range of validity of $H_{\mathrm{D}}$. However below $\Gamma_{\mathrm{m}}, H_{\mathrm{D}}$ should be compared with the sum of isolated dislocations and of disclination pairs that appear close to the transition.

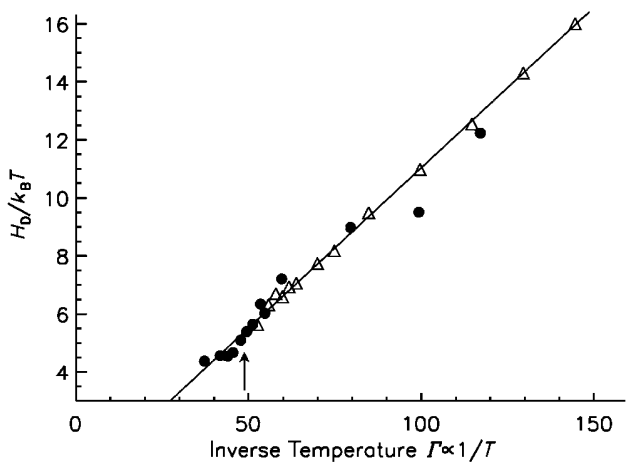

Figure 9. $\Gamma$-dependence of the dislocation interaction for dislocations at minimum distance $R / a_{0}=1$ and antiparallel Burgers vectors, determined by experiment $(\bullet)$ and simulation $(\triangle)$. The behavior expected from $H_{\mathrm{D}}$ [Eq. (1)] is shown by the solid line. The melting point $\Gamma_{\mathrm{m}}$ is indicated by the arrow. Reprinted with permission from ref. [14]. Copyright 2005 by the American Physical Society.
A deviation between $H_{D}$ and the results from experiments and MC simulations is found for the angular dependence of the dislocation interaction. The comparison shown in Figure 10

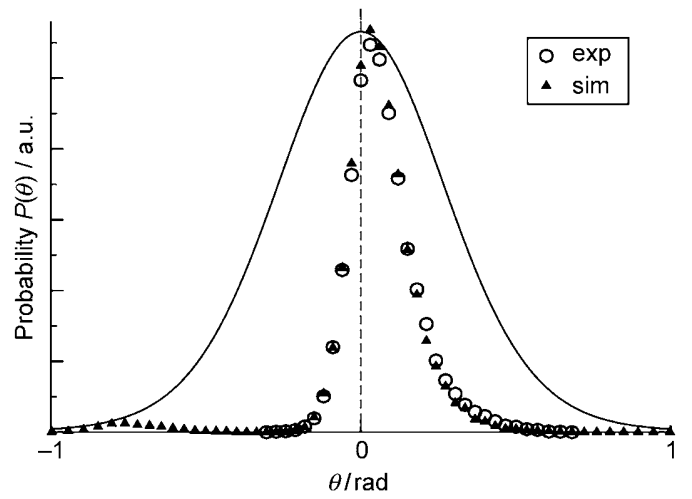

Figure 10. Distribution of the angle $\theta$ between Burgers vector $\vec{b}$ and the vector $\vec{R}$ joining the two dislocations in a pair at minimum distance $R=a_{0}$ and with antiparallel Burgers vectors $\vec{b}_{1}=-\vec{b}_{2}$. The line shows the distribution expected from the Hamiltonian $H_{D}$ [Eq. (1)]. Reprinted with permission from ref. [14]. Copyright 2005 by the American Physical Society.

was determined for dislocations at minimum distance and antiparallel Burgers vectors at $\Gamma=55$. This difference between the distribution $P(\theta)=\exp \left[Y a_{0}^{2} \beta \cos ^{2} \theta /(4 \pi)\right]$ expected from theory and the experimental results is due to the microscopic scale of the experimental data, which is not included in the continuum approach of the theory. The $\theta$-distribution from experiment is asymmetric, because a negative $\theta$-value corresponds to the sevenfold coordinated particles of the pair moving further away from each other, which leads to the annihilation of the pair as can be seen in Figure 1 B.

As the Hamiltonian $H_{\mathrm{D}}$ is one of the starting points of KTHNY theory, its generally good agreement with experimental results is a further corroboration of the melting behavior according to KTHNY in the colloidal model system studied.

\section{Anisotropic Interaction}

The evidence for crystal melting according to KTHNY theory as presented in the preceding sections raises the question whether this melting scenario is robust with respect to changes of the system. This has been studied for the case of an externally imposed anisotropy of the particle interaction, which is obtained by tilting the external magnetic field away from the perpendicular direction. The effect on the melting behavior of the crystal can be expected to be important, since the crystalline structure and the dynamics of the particles are observed to change. Therefore, it can be expected that the phase behavior is also affected and whether new pathways for melting preempt the KTHNY scenario should be expected to depend on the details of the anisotropy. The case of an anisotropic interaction due to elongated particles was studied by Ostlund, Halperin, Toner, and Nelson. ${ }^{[38,39]}$ They found that in the presence of a symmetry axis, such as due to anisotropy, there are two types of dislocations-one type with Burgers vector along the symmetry axis (type I) and another with Burgers vector at an 
angle $\pm \phi$ from the axis (type II). Depending on the nature of the anisotropy, they predicted that one type of dislocation would unbind first. For the preferred unbinding of type I they found that the crystal should melt into a 2D smectic phase.

Herein, we review the effect of a uniform, externally imposed anisotropy, which is obtained in the colloidal model system introduced in Section 4 by tilting the external magnetic field by an angle $\varphi$ away from the direction perpendicular to the particle plane. The interaction between the particles becomes anisotropic [Eq. (10)]: ${ }^{[36]}$

$u(r)=\frac{\mu_{0}(\chi B)^{2}}{8 \pi} \frac{\left(1-3 \sin ^{2} \varphi \cos ^{2} \theta\right)}{r^{3}}$

where $\cos \theta=\vec{r} \cdot \vec{B}_{\|} /\left(|\vec{r}|\left|\vec{B}_{\|}\right|\right)$. The tilting of the field reduces the repulsion along the in-plane component $\vec{B}_{\|}$of the magnetic field and the particles can move closer to each other along this direction. For tilting angles $\varphi \geq 10^{\circ}$ the anisotropy is strong enough to orient the crystal. As shown in Figure $11 \mathrm{~B}$, the unit cell is no longer triangular but becomes oblique or,

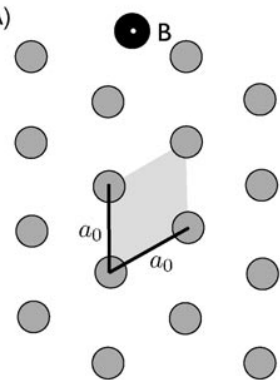

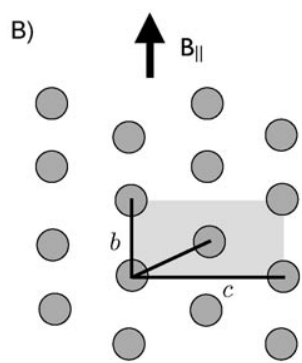

Figure 11. A) Hexagonal lattice with lattice constant $a_{0}$ for isotropic interaction with the external magnetic field $\vec{B}$ perpendicular to the particle plane. The primitive unit cell is highlighted by the grey area. B) Rectangular lattice as observed for anisotropic interaction with an in-plane magnetic component $\vec{B}_{\|}$. The unit cell containing two particles is shown by the grey area.

equivalently, centered rectangular with lattice constants $b$ and $c$ and each particle has two nearest neighbors that lie in the direction of $\vec{B}_{\|}$and four second nearest neighbors are situated on adjacent lattice lines oriented along $\vec{B}_{\|}$. The ratio $b / c$ of the lattice constants was measured for various tilting angles $\varphi^{[40]}$ and agrees very well with the expectation from harmonic lattice theory. ${ }^{[41]}$

The behavior of the Lindemann parameter [Eq. (3)] and the correlation functions $G_{T}(r)$ and $G_{6}(r)$ [Eqs. (2) and (4)] can be compared directly to the case of isotropic interaction $\left(\varphi=0^{\circ}\right)$ by rescaling the lattice constant $b$ shown in Figure $11 \mathrm{~B}$ such that the triangular lattice is recovered.

\subsection{Weak Anisotropy}

For tilting angles $\varphi<22^{\circ}$, a rescaling of $b$ to the isotropic case shows that the behavior of the Lindemann parameter is unchanged. Without rescaling the particle deviations are larger in the direction perpendicular to $\vec{B}_{\|}$, as expected from harmonic lattice theory. ${ }^{[42]}$ In analogy to the behavior of $\gamma_{L}(t)$, the melting transition keeps the same character: As shown in
Figure 12, the effective melting temperature remains almost unchanged for tilting angles $\varphi<22^{\circ}$. The crystal melts into the quasi-hexatic phase, which has essentially the same prop-

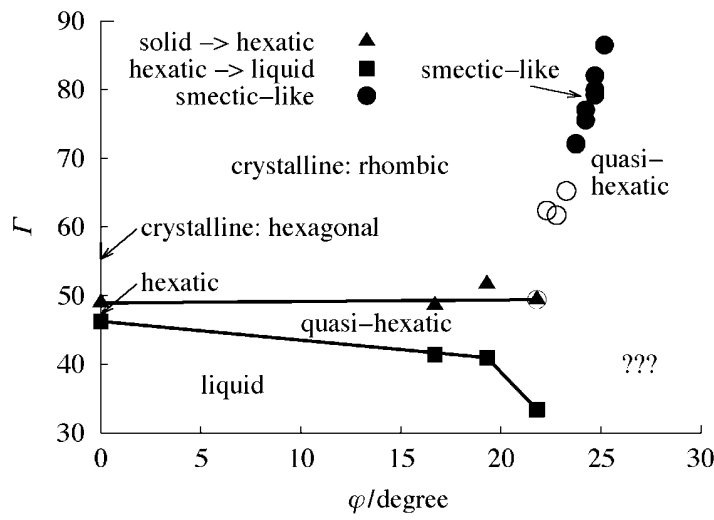

Figure 12. Phase diagram of a 2D system of particles with magnetic dipoledipole interaction. Inverse temperature $\Gamma$ is shown on the $y$-axis and the anisotropy of the interaction represented by the tilting angle of the external magnetic field is shown on the $x$-axis.

erties as the hexatic, but due to the distortion of the crystal lattice the orientational symmetry is not strictly sixfold as the word 'hexatic' would suggest. The anisotropy causes $\Gamma_{i}$ to decrease considerably (Figure 12). This stabilization of the quasihexatic phase is caused by the tilted external field, which imposes a preferred direction and strengthens the orientational order.

\subsection{Strong Anisotropy}

For tilting angles $\varphi \geq 22^{\circ}$, the behavior of the Lindemann parameter is no longer explained by a rescaling of the lattice constant $b$. The deviations along $\vec{B}_{\|}$become larger than in the perpendicular direction. ${ }^{[41]}$ This cross-over of $\gamma_{L, \|}(t)$ and $\gamma_{L, \perp}(t)$ reflects the reduced dipole-dipole repulsion along the in-plane component of the magnetic field. The fast increase of $\gamma_{L, \|}(t)$ with $\varphi$ indicates the proximity of a soft mode of lattice vibrations in this direction. ${ }^{[42]}$ The larger fluctuations along the parallel direction lead to an enhanced formation of dislocations, which are oriented such that they reduce the translational order along $\vec{B}_{\|}$. Indeed, for $\varphi>22^{\circ}$ the crystal is destabilized considerably and the melting behavior as reflected by $G_{T}(r)$ becomes anisotropic. ${ }^{[43]}$ The crystal melts just along the parallel direction at much higher inverse temperatures in the range $72<\Gamma<82$. Accordingly, $G_{\mathrm{T}}(r)$ shows an exponential decay along $\vec{B}_{\|}$and an algebraic decay due to quasi-long-range order along $\vec{B}_{\|}$(Figure 13). The Lindemann parameter $\gamma_{L}(t)$ shows the same behavior. It approaches a constant value for fluctuations perpendicular $\vec{B}_{\|}$but diverges for fluctuations along the $\vec{B}_{\|}$-direction. As expected from refs. $[38,39]$, the system melts into a columnar phase (see Figure 12) and the type II dislocations with the Burgers vector oriented at a finite angle with respect to $\vec{B}_{\|}$are strongly suppressed. ${ }^{[43]}$ Furthermore, dislocation pairs formed by dislocations with the Burgers vector perpendicular to $\vec{B}_{\|}$are completely suppressed. This reflects the 


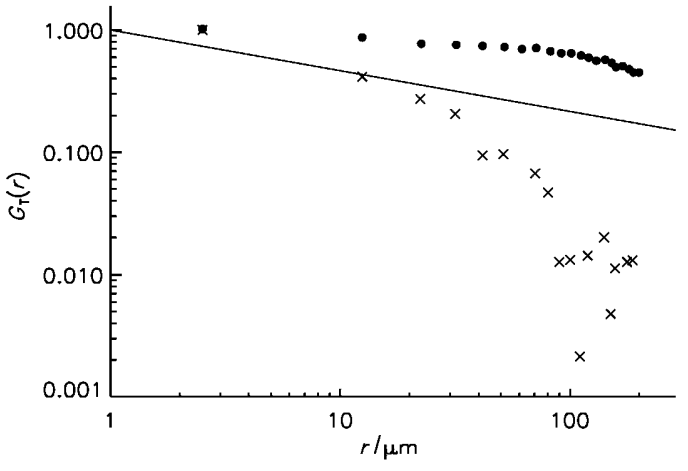

Figure 13. Translational correlation function for a reciprocal lattice vector parallel $(\times)$ and perpendicular $(\bullet)$ to $\vec{B}_{\|}$obtained at a tilting $\varphi=24.2^{\circ}$ and $\Gamma=76$. The line shows the decay $\propto r^{-1 / 3}$ as expected at melting with isotropic interaction. Reprinted with permission from ref. [43]. Copyright 2004 by the American Physical Society.

strong localization of the particles in columns due to the strong repulsion perpendicular to $\vec{B}_{\|}$. Thus, two particles belonging to adjacent columns cannot come close enough to become the sevenfold coordinated particles of a dislocation pair.

\section{Conclusions}

An experimental confirmation for the KTHNY theory for crystal melting in 2D has been found with the presented colloidal model system with repulsive magnetic dipole-dipole interaction. Thus, KTHNY is expected to be relevant for other 2D systems in which these underlying topological defects can form. However, clear criteria for this class of 2D crystals are currently missing. The core energy of dislocations plays an essential role for this question, as it must be low enough to allow their spontaneous appearance. Furthermore, the presented experiments indicate that KTHNY theory is robust with respect to a transition from isotropic to anisotropic interaction as long as dislocations with all orientations can form. Upon the suppression of a type of dislocation the KTHNY scenario changes. The crystal has been found to melt into a columnar instead of the hexatic phase for sufficiently strong anisotropy of the interaction.

\section{Acknowledgements}

U. G. acknowledges financial support from the Adolphe Merkle Foundation. This work has been supported by the Deutsche Forschungsgemeinschaft (DFG) by means of the SFB TR 6 and SFB 513 programs and by an International Research Training Group of the German French University.

Keywords: anisotropy · colloids · crystal growth · throughspace interactions $\cdot$ two-dimensional crystals

[1] A. P. Young, Phys. Rev. B 1979, 19, 1855-1866.
[2] D. R. Nelson, B. I. Halperin, Phys. Rev. B 1979, 19, 2457-2484.

[3] J. M. Kosterlitz, D. J. Thouless, J. Phys. C 1973, 6, 1181-1203.

[4] K. J. Strandburg, Rev. Mod. Phys. 1988, 60, 161-207.

[5] D. R. Nelson, Defects and Geometry in Condensed Matter Physics, Cambridge University Press, Cambridge, 2002.

[6] H.-H. von Grünberg, P. Keim, G. Maret in Soft Matter, vol. 3, Colloidal Order from Entropic and Surface Forces (Eds: G. Gompper, M. Schick), Wiley-VCH, Weinheim, 2007, pp. 40-83.

[7] R. Peierls, Ann. I. H. Poincare 1935, 5, 177-222.

[8] A. M. Alsayed, M. F. Islam, J. Zhang, P. J. Collings, A. G. Yodh, Science 2005, 309, 1207-1210.

[9] S. T. Chui, Phys. Rev. Lett. 1982, 48, 933-935.

[10] S. T. Chui, Phys. Rev. B 1983, 28, 178-194.

[11] H. Kleinert, Phys. Lett. A 1983, 95, 381-384.

[12] M. A. Glaser, N. A. Clark in Advances in Chemical Physics, Vol. 83 (Eds.: I. Prigogine, S. A. Rice), John Wiley and Sons, New York, 1993, pp. 543709.

[13] F. R. N. Nabarro, Theory of Crystal Dislocations, Dover Publications, New York, 1987.

[14] C. Eisenmann, U. Gasser, P. Keim, G. Maret, Phys. Rev. Lett. 2005, 95, 185502.

[15] F. A. Lindemann, Phys. Z. 1910, 14, 609-612.

[16] V. M. Bedanov, G. V. Gadiyak, Y. E. Lozovik, Phys. Lett. A 1985, 109, 289291.

[17] K. Zahn, R. Lenke, G. Maret, Phys. Rev. Lett. 1999, 82, 2721 - 2724.

[18] M. J. Stephen, J. P. Straley, Rev. Mod. Phys. 1974, 46, 617-704.

[19] F. Moučka, I. Nezbeda, Phys. Rev. Lett. 2005, 94, 040601.

[20] K. Chen, T. Kaplan, M. Mostoller, Phys. Rev. Lett. 1995, 74, 4019-4022.

[21] F. L. Somer, Jr., G. S. Canright, T. Kaplan, L. Chen, M. Mostoller, Phys. Rev. Lett. 1997, 79, $3431-3434$.

[22] A. Jaster, Phys. Rev. E 1999, 59, 2594-2602.

[23] S. Z. Lin, B. Zheng, S. Trimper, Phys. Rev. E 2006, 73, 066106.

[24] C. A. Murray, D. H. van Winkle, Phys. Rev. Lett. 1987, 58, 1200-1203.

[25] Y. Tang, A. J. Armstrong, R. C. Mockler, W. J. Osullivan, Phys. Rev. Lett. $1989,62,2401-2404$

[26] A. H. Marcus, S. A. Rice, Phys. Rev. Lett. 1996, 77, 2577-2580.

[27] R. E. Kusner, J. A. Mann, J. Kerins, A. J. Dahm, Phys. Rev. Lett. 1994, 73, $3113-3116$.

[28] R. E. Kusner, J. A. Mann, A. J. Dahm, Phys. Rev. B 1995, 51, 5746-5759.

[29] K. Zahn, G. Maret, Phys. Rev. Lett. 2000, 85, 3656-3659.

[30] P. Keim, G. Maret, H. H. von Grünberg, Phys. Rev. E 2007, 75, 031402.

[31] K. Zahn, J. M. Mendez-Alcarez, Phys. Rev. Lett. 1997, 79, 175-178.

[32] F. Ebert, P. Dillmann, G. Maret, P. Keim, Rev. Sci. Instrum. 2009, 80, 083902.

[33] H. H. von Grünberg, P. Keim, K. Zahn, G. Maret, Phys. Rev. Lett. 2004, 93, 255703.

[34] N. D. Mermin, H. Wagner, Phys. Rev. Lett. 1966, 17, 1133-1136.

[35] P. Keim, G. Maret, U. Herz, H. H. von Grünberg, Phys. Rev. Lett. 2004, 92, 215504

[36] H. Loewen, R. Messina, N. Hoffmann, C. N. Likos, C. Eisenmann, P. Keim, U. Gasser, G. Maret, R. Goldberg, T. Palberg, J. Phys. Condens. Mat. 2005 17, S3379-S3386

[37] P. Dillmann, G. Maret, P. Keim, J. Phys. Condens. Matter 2008, 20, 404216.

[38] S. Ostlund, B. I. Halperin, Physis Physical Review B 1981, 23, 335-358.

[39] J. Toner, D. R. Nelson, Physis Physical Review B 1981, 23, 316-334.

[40] C. Eisenmann, P. Keim, U. Gasser, G. Maret, J. Phys. Condens. Matter 2004, 16, S4095.

[41] V. A. Froltsov, R. Blaak, C. N. Likos, H. Löwen, Phys. Rev. E 2003, 68, 061406.

[42] V. A. Froltsov, C. N. Likos, H. Löwen, C. Eisenmann, U. Gasser, P. Keim, G. Maret, Phys. Rev. E 2005, 71, 031404

[43] C. Eisenmann, U. Gasser, P. Keim, G. Maret, Phys. Rev. Lett. 2004, 93, 105702.

[44] J. Zanghellini, P. Keim, H. H. von Grünberg, J. Phys. Condens. Matter 2005, 17, S3579-S3586.

Received: September 25, 2009

Published online on January 22, 2010 